\title{
Importancia del estado de flujos de efectivo para la gestión financiera sostenible*
}

\author{
Importance of the cash flow statement for sustainable financial management \\ Importância da demonstração dos fluxos de caixa para uma gestão financeira sustentável
}

Jaime Andrés Correa-Garcia ${ }^{\text {a }}$

Universidad de Antioquia, Colombia

DOI: https://doi.org/10.11144/Javeriana.cc22.iefe

jaime.correa@udea.edu.co

ORCID: https://orcid.org/0000-0001-8814-2107

Recibido: 04/08/2020

Diego Andrés Correa-Mejía

Aceptado: $22 / 09 / 2021$

Universidad de Antioquia, Colombia

Publicado: 31/12/2021

ORCID: https://orcid.org/0000-0002-1319-0451

\section{Resumen:}

El propósito de este artículo es evidenciar las prácticas de presentación del Estado de Flujos de Efectivo (EFE) y los aportes que brinda para la gestión financiera sostenible. Este trabajo se fundamenta en la alta aplicación que tienen los flujos de caja en las finanzas corporativas y en el creciente interés por el EFE a raíz de la reciente implementación de las Normas Internacionales de Información Financiera (NIIF) en Colombia. Apoyados en una muestra de 14.979 empresas, se exponen ocho escenarios de interpretación y análisis basados en el EFE y el Flujo de Caja Libre (FCL) para su aplicación empresarial. En este estudio se aplicó un análisis correlacional multivariado a través del método Biplot que arrojó como resultado que las empresas que generan utilidades netas positivas no necesariamente cuentan con flujos de caja que les permita atender sus acreencias financieras y dividendos. Este trabajo aporta a la literatura académica sobre el estado de flujos de efectivo y sienta las bases para trabajos futuros una vez se consoliden estas nuevas normas en Colombia.

Códigos JEL: G30, M41, M49.

Palabras clave: Estado de flujos de efectivo, gestión financiera sostenible, flujo de caja libre, NIIF.

\section{Abstract:}

The purpose of this paper is to present the importance of the cash flow statement (CFS) for sustainable financial management. This study is based on the high application of cash flow in corporate finance and the growing interest in the CFS as a result of the implementation of International Financial Reporting Standards (IFRS) in Colombia. A sample of 14,979 companies was used to illustrate several interpretation and analysis scenarios, based on the CFS and Free Cash Flow (FCF), for business application. In this study, a multivariate correlational analysis was applied through the Biplot method. This method meant it was possible to show that companies that generate positive net profits do not necessarily have sufficient cash flow to allow them to pay financial claims and dividends. This work contributes to the academic literature on the cash flow statement and presents an advance for future work once new standards are consolidated in Colombia.

JEL Codes: G30, M41, M49.

Keywords: Cash flow statement, sustainable financial management, free cash flow, IFRS.

\section{Resumo:}

O objetivo deste artigo é evidenciar as práticas de apresentação da Demonstração dos Fluxos de Caixa (DFC) e as contribuições que ela proporciona para uma gestão financeira sustentável. Este trabalho se baseia na alta aplicação de fluxos de caixa em finanças corporativas e no crescente interesse pela DFC como resultado da implementação das Normas Internacionais de Relatório Financeiro (International Financial Reporting Standards - IFRS) na Colômbia. Com base em uma amostra de 14.979 empresas, oito cenários de interpretação e análise baseados na DFC e no Fluxo de Caixa Livre (FCL) são expostos para sua aplicação comercial. Neste estudo, foi aplicada uma análise correlacional multivariada por meio do método Biplot, que mostrou que as empresas que geram lucros líquidos positivos não necessariamente possuem fluxos de caixa que lhes permitam pagar suas dívidas financeiras e dividendos. Este trabalho contribui para a literatura acadêmica sobre a demonstração dos fluxos de caixa e estabelece as bases para trabalhos futuros, uma vez que esses novos padrões sejam consolidados na Colômbia.

Códigos JEL: G30, M41, M49.

Notas de autor

\footnotetext{
a Autor de correspondencia. E-mail: jaime.correa@udea.edu.co
} 
Palavras-chave: Demonstração dos fluxos de caixa, gestão financeira sustentável, fluxo de caixa livre, IFRS.

\section{Introducción}

Este estudio analiza la presentación del Estado de Flujos de Efectivo (EFE) bajo las Normas Internacionales de Información Financiera (NIIF) en Colombia durante el año 2018 con el objetivo de evidenciar las prácticas de presentación de este estado financiero, así como los aportes que brinda para la gestión financiera sostenible. Realizamos este trabajo considerando la reciente aplicación de las NIIF en Colombia y debido a las falencias en la presentación del EFE evidenciadas por Correa, Pulgarín, Muñoz et al. (2010) y al poco uso que se le da al mismo en la gestión financiera empresarial en esta economía emergente.

El EFE además de ser un estado financiero de propósito general que cumple el papel de informar sobre la gestión y uso del efectivo, es una valiosa herramienta para el análisis financiero (Correa-Mejía \& LoperaCastaño, 2020; Estridge \& Lougee, 2007) y la toma de decisiones empresariales (Zhang, 2019). Es por esto que ante la reciente aplicación de las NIIF en Colombia estudiamos las prácticas de presentación del EFE e identificamos los resultados agregados para diversos sectores de la economía que reportan información a la Superintendencia de Sociedades. Con la base de datos para empresas no cotizadas analizamos los riesgos de continuidad empresarial y sostenibilidad financiera siguiendo a Terreno et al. (2020) quienes de manera similar a Sayari \& Mugan (2013) aplican una taxonomía que divide los resultados de las empresas en ocho escenarios. Estos modelos permiten conceptuar sobre la salud financiera corporativa a partir de la gestión del efectivo.

Para el análisis de los resultados del EFE incorporamos las alternativas posibles en la estructura de este estado financiero que plantean las NIIF (particularmente la NIC 7) para los intereses recibidos, dividendos recibidos e intereses pagados. Estas reclasificaciones fueron incorporadas en los análisis y recogen la flexibilidad que permite la referida norma (Gordon, Henry, Jorgensen et al., 2017). De esta manera los intereses y dividendos recibidos fueron clasificados en el flujo de efectivo por actividades de inversión y los intereses pagados fueron clasificados en el flujo de efectivo por actividades de financiación, lo anterior reconociendo la actividad que origina estos movimientos de efectivo.

Considerando la importancia del Flujo de Caja Libre (FCL) en el análisis financiero y la toma de decisiones empresariales tal como lo plantean Shyam \& Mollie (2017), incluimos el análisis de las variables cobertura combinada con el propósito de conceptuar con base en ellas sobre los riesgos de continuidad y la capacidad de gestión financiera sostenible de las empresas que reportaron información de sus flujos de efectivo bajo NIIF. La cobertura combinada evalúa la capacidad de pago del servicio de la deuda y los dividendos a partir del FCL (Correa-Garcia, 2007). Es de anotar que metodológicamente construimos el FCL a partir del propio EFE aportando así evidencia empírica de cómo se puede hacer la transición del estado financiero de propósito general (EFE) a este informe financiero de propósito especial (FCL) que tiene amplias y variadas aplicaciones en el campo de las finanzas corporativas (Kousenidis, 2006).

Para el análisis de los resultados se hizo uso del modelo biplot desarrollado por Gabriel (1971). A través de este modelo fue posible realizar un análisis multivariado y correlacional de las variables del EFE y FCL con la sostenibilidad financiera, mostrando que la capacidad de pago con acreedores financieros y accionistas está positivamente correlacionada con la generación de efectivo a partir de la operación.

Esta investigación aporta en dos vías: la primera está relacionada con el campo práctico ya que el análisis de la presentación actual del EFE de diversas empresas de Colombia, evidencia las diferentes fortalezas y debilidades más comunes en la presentación y revelación de los estados financieros. La segunda se encuentra relacionada con la academia, toda vez que se suministra información que se debe considerar en la elaboración del EFE y la forma como se debe hacer gestión para lograr la sostenibilidad financiera apoyados en los flujos de efectivo, lo cual está en línea con lo planteado por Duque (2015) sobre la importancia del EFE en la formación 
de los contadores públicos. Nuestra contribución a la literatura y la aplicación gerencial se sustenta en que trabajos previos como Terreno et al. (2017), Duque (2015) y Sayari \& Mugan (2013) se han centrado en la forma de presentación y análisis, pero no en un ambiente bajo NIIF. Adicionalmente evidenciar la estrecha relación entre el EFE y FCL es de gran utilidad para los tomadores de decisiones y académicos que utilizan estas herramientas con distintos propósitos en la gestión empresarial.

\section{Marco de referencia}

Este marco referencial presenta la evolución normativa del Estado de Flujos de Efectivo en Colombia y un apartado sobre la aplicabilidad de este estado financiero en la gestión empresarial.

\section{Etapas de la regulación de los estados financieros en Colombia}

Los estados financieros cumplen el papel de informar a los distintos usuarios de la información contable (Chen \& Gong, 2019) sobre la posición financiera (ESF), el desempeño de la entidad (ERI), los cambios patrimoniales (ECP) y sobre la capacidad de generación y gestión del efectivo (EFE). Los estados financieros además de ser un instrumento de comunicación son un mecanismo para la gestión empresarial y la toma de decisiones (Agudelo-Rodríguez, Flórez-Londoño, \& Correa-Mejía, 2020; Charitou, Karamanou, \& Kopita, 2018). Cada uno de ellos cumple funciones específicas, por lo cual su lectura y análisis debe ser integral para ayudar de manera más efectiva a una gestión financiera sostenible, es decir con una orientación de largo y que no se limite sólo a informar el pasado y cumplir con requerimientos legales.

En Colombia no siempre han sido obligatorios estos cuatro estados financieros, por lo cual su uso y requerimiento por las distintas entidades de vigilancia y control ha variado en el tiempo (Sierra, 2001). El avance de la regulación contable en el contexto colombiano se puede evidenciar en cuatro etapas que han impactado directamente a los estados financieros de acuerdo con lo señalado por Correa et al. (2010). La última fase se presenta a partir de la Ley 1314 de 2009 con la cual se presentó la implementación de las NIIF y se dividieron las empresas en tres grupos de aplicación. La tabla 1 resume esta evolución.

TABLA 1

Regulación de los estados financieros en Colombia

\begin{tabular}{|c|l|l|c|}
\hline Etapa & \multicolumn{1}{|c|}{ Marco normativo } & \multicolumn{1}{|c|}{ Estados financieros } & $\begin{array}{c}\text { Flujo de } \\
\text { efectivo }\end{array}$ \\
\hline 1 & $\begin{array}{l}\text { Decreto } 410 \text { de } 1971 \\
\text { (Código de Comercio) }\end{array}$ & $\begin{array}{l}\text { Balance general } \\
\text { Estado de pérdidas y ganancias }\end{array}$ & No \\
\hline \multirow{2}{*}{3} & Decreto 2160 de 1986 & $\begin{array}{l}\text { Balance general } \\
\text { Estado de resultados } \\
\text { Estado de ganancias (déficit) acumulado } \\
\text { Estado de cambios en la situación financiera }\end{array}$ & No \\
\hline \multirow{2}{*}{3} & $\begin{array}{l}\text { Balance general } \\
\text { Estado de resultados } \\
\text { Estado de cambios en el patrimonio } \\
\text { Estado de cambios en la situación financiera } 2649 \text { de 1993 } \\
\text { Estado de flujos de efectivo }\end{array}$ & $\mathrm{Si}$ \\
\cline { 2 - 5 } & $\begin{array}{l}\text { Ley 222 de 1995 } \\
\text { Para estados financieros } \\
\text { consolidados }\end{array}$ & $\begin{array}{l}\text { Balance general } \\
\text { Estado de resultados } \\
\text { Estado de cambios en el patrimonio } \\
\text { Estado de flujos de efectivo }\end{array}$ & $\mathrm{Si}$ \\
\hline \multirow{2}{*}{4} & $\begin{array}{l}\text { Decreto 2420 de 2015 } \\
\text { Grupo 1 (NIIF Plenas) y } \\
\text { Grupo 2 (NIIF PYMES) }\end{array}$ & $\begin{array}{l}\text { Estado de situación financiera } \\
\text { Estado de resultados integrales } \\
\text { Estado de cambios en el patrimonio } \\
\text { Estado de flujos de efectivo }\end{array}$ & $\mathrm{Si}$ \\
\cline { 2 - 5 } & $\begin{array}{l}\text { Decreto 2420 de 2015 } \\
\text { Grupo 3 }\end{array}$ & $\begin{array}{l}\text { Estado de situación financiera } \\
\text { Estado de resultados }\end{array}$ & No \\
\hline
\end{tabular}

Fuente: elaboración propia.

Como se observa en la tabla 1, el EFE ha estado presente en el contexto colombiano desde 1994, lo que genera la posibilidad a las empresas de realizar gestión de sus flujos de caja con el fin de lograr la sostenibilidad 
financiera y la generación de valor (Yaari, Nikiforov, Kahya et al., 2016). Sin embargo, la cultura contable y empresarial colombiana no ha sido proclive hacia el uso gerencial del EFE y éste se ha limitado principalmente a cumplir requerimientos legales (Correa, 2005). Por el contrario, los otros flujos de caja son de gran ayuda en la gestión financiera empresarial, por lo cual tienen gran acogida. En definitiva, queremos plantear una sub-utilización del EFE como informe contable y generar alternativas de uso de este estado financiero como herramienta para la gestión financiera.

\section{Gestión financiera a partir del Estado de Flujos de Efectivo}

El EFE es el único estado financiero de propósito general base caja (Bradbury, 2011) y que tiene el propósito de presentar información financiera relacionada con los recaudos y desembolsos de efectivo que las compañías han experimentado durante un periodo de tiempo específico. Adicionalmente, el EFE es un estado financiero dinámico (marginal) ya que combina información del ERI (dinámico) y del ESF (estático). Los movimientos de efectivo que se muestran a través del estado financiero se deben clasificar en flujos de efectivo por actividades de operación, inversión y financiación arrojando así luces sobre las decisiones de la empresa (Vishwanath \& Krishnamurti, 2009). Esta taxonomía está en sintonía con las principales decisiones de estudio en el campo de las finanzas corporativas que son las decisiones de operación, inversión, financiación y dividendos (Rivera-Godoy \& Rendón-Perea, 2019; Mascareñas, 2013), con lo cual se puede advertir que el EFE más que ser un estado financiero de carácter legal, tiene el potencial de facilitar la gestión financiera sostenible.

El flujo de efectivo de operación ha contado desde sus inicios con dos métodos de presentación: el método directo y el método indirecto. El primero consiste en mostrar directamente los recaudos y pagos derivados del desarrollo del objeto social que tuvo una compañía durante un periodo determinado (Bradbury, 2011). Con ese método -de cierta manera- se busca presentar el estado de resultados como si fuera de caja. El segundo, según Orpurt \& Zang (2007) también es conocido como el método de conciliación de la utilidad, ya que se parte de la utilidad (operacional o neta) y se depura con los rubros que afectaron el estado de resultados, pero que no implicaron movimiento directo de efectivo. Posteriormente se asocia también a esta utilidad corregida, el efecto que genera la gestión financiera de corto plazo recogido en el capital de trabajo neto (variación) (Baños-Caballero, García-Teruel, \& Martínez-Solano, 2014). Los flujos de inversión y financiación se presentan identificando las entradas y salidas por estos componentes (Hales \& Orpurt, 2013). Independiente del método de presentación del estado de flujos de efectivo, el esquema general del EFE no varía y es el que se muestra en la figura 1 . 


\begin{tabular}{|l|}
\hline Efectivo por Actividades de Operación (EAO) \\
\hline Efectivo por Actividades de Inversión (EAI) \\
\hline Efectivo por Actividades de Financiación (EAF) \\
\hline $\begin{array}{l}\text { (=) Variación del efectivo y equivalentes } \\
(+) \text { Saldo inicial } \\
\text { (=) Saldo final }\end{array}$ \\
\hline
\end{tabular}

FIGURA 1

Esquema del Estado de Flujos de Efectivo

Fuente: elaboración propia.

Las NIIF permiten alternativas de clasificación para algunos rubros espaciales tal como fue analizado por Gordon et al. (2017) con una muestra internacional de este estado financiero para los intereses pagados, intereses recibidos y dividendos recibidos. Los ingresos financieros (incluyendo los dividendos recibidos) que no tengan relación alguna con la operación se pueden (deben) incorporar en las actividades de inversión ya que son las inversiones las que generan los intereses que reciben las empresas (Baik, Cho, Choi et al., 2016). Por su parte, los gastos financieros que no se deriven de actividades operacionales de las compañías, se pueden (deben) presentar dentro de los flujos de financiación, ya que las obligaciones financieras adquiridas por las compañías son las que generan el pago de intereses que se realizan (Charitou et al., 2018). Adicionalmente, la NIC 7 establece que los impuestos a las ganancias se deberían separar entre las actividades de operación, inversión y financiación de acuerdo con la transacción que haya originado el impuesto respectivo. En la figura 2 se muestra la clasificación de las partidas especiales del estado de flujos de efectivo.

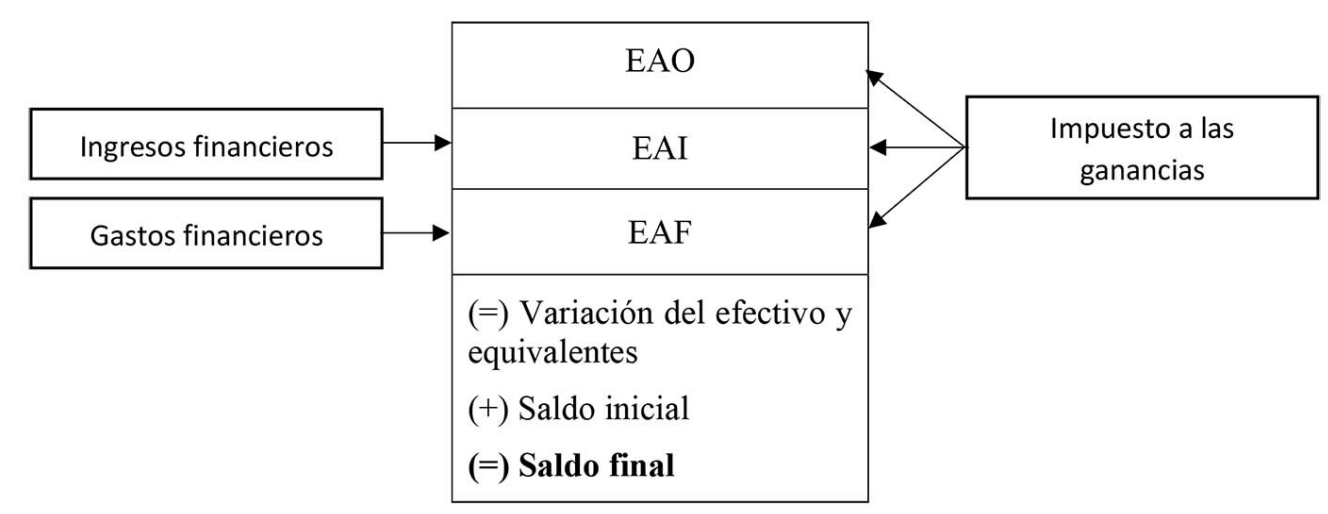

FIGURA 2

Partidas especiales en el Estado de Flujos de Efectivo Fuente: elaboración propia.

Como lo muestra la figura 2, el EFE explica el efectivo y sus equivalentes, permitiendo identificar la actividad específica de donde se están obteniendo los flujos de efectivo y las actividades que se están consumiendo este recurso. 
En consonancia con Sayari \& Mugan (2013) el análisis del EFE permite evidenciar riesgos de continuidad cuando se observan flujos operacionales negativos y flujos de inversión positivos (desinversión de activos) de manera recurrente. La existencia de flujos de financiación periódicamente positivos, también puede advertir potenciales riesgos si esta situación obedece al impago de deudas o al crecimiento desmedido de pasivos financieros. Es con el análisis de situaciones como estas que el flujo de caja cobra relevancia en el quehacer organizacional. Asimismo el trabajo de modelo factorial para el análisis de los flujos de caja de Soboleva, Matveev, Ilminskaya et al. (2018) evidencia la importancia de esta herramienta para la identificación oportuna de riegos financieros y por tanto de la sostenibilidad financiera de la entidad. Por otro lado, Kroes \& Manikas (2014) analizan factores específicos del flujo de caja y estudian su incidencia en el desempeño financiero con lo cual se evidencia empíricamente la posibilidad que establece la gestión basada en flujos de caja para el establecimiento de estrategias financieras que garanticen un buen desempeño y sostenibilidad en el largo plazo.

Si bien el EFE por sí mismo puede contribuir a la toma decisiones, es importante considerar también la articulación que existe entre el EFE y el Flujo de Caja Libre (FLC) (Correa, Martínez, Ruiz et al., 2018). Como variante del EFE, el FCL tiene como objetivo presentar de manera clara la generación operacional de efectivo al interior de la empresa y la manera como es destinado a los proveedores del capital financiero. El FCL es una variable fundamental para la gestión financiera sostenible de cualquier empresa porque con su ayuda es posible monitorear la generación de valor de las empresas y de acuerdo con Correa-Garcia (2007) gestionar financieramente a las empresas con el fin de ser sostenibles en el tiempo. La sostenibilidad financiera por medio de los FCLO (FCL Operacional) se logra cuando estos son positivos (la empresa ya cubrió todos sus compromisos operacionales: costos, gastos e impuestos operativos, CTNO y CAPEX) y alcanzan a cubrir los pagos a terceros (servicio de la deuda) y el pago a los accionistas (dividendos). De esta manera el FCL plantea una respuesta en el marco de la teoría de la agencia (Jensen, 1986) y propone un modelo de análisis orientado a la sostenibilidad de largo plazo, ya que éste incluye integralmente la toma de decisiones de cara a la creación de valor. Esto se debe a la importancia que tienen los flujos de caja en el proceso de creación de valor empresarial y por lo tanto como los agentes responden a los principales sobre ese propósito principal para el que son contratados y es la sostenibilidad empresarial y la generación de valor. De esta manera desde un punto de vista financiero se contribuye a la solución a uno de los problemas de agencia que identifica Bendickson, Muldoon, Liguori et al. (2016) asociado a la asimetría de información y las diferentes posiciones frente al riesgo.

Si bien el FCL no es considerado bajo la normatividad como un estado financiero, la estructura de este se deriva de los estados financieros principalmente del EFE. La figura 3 muestra la relación existente entre el flujo de efectivo bajo NIC 7 y el flujo de caja libre.

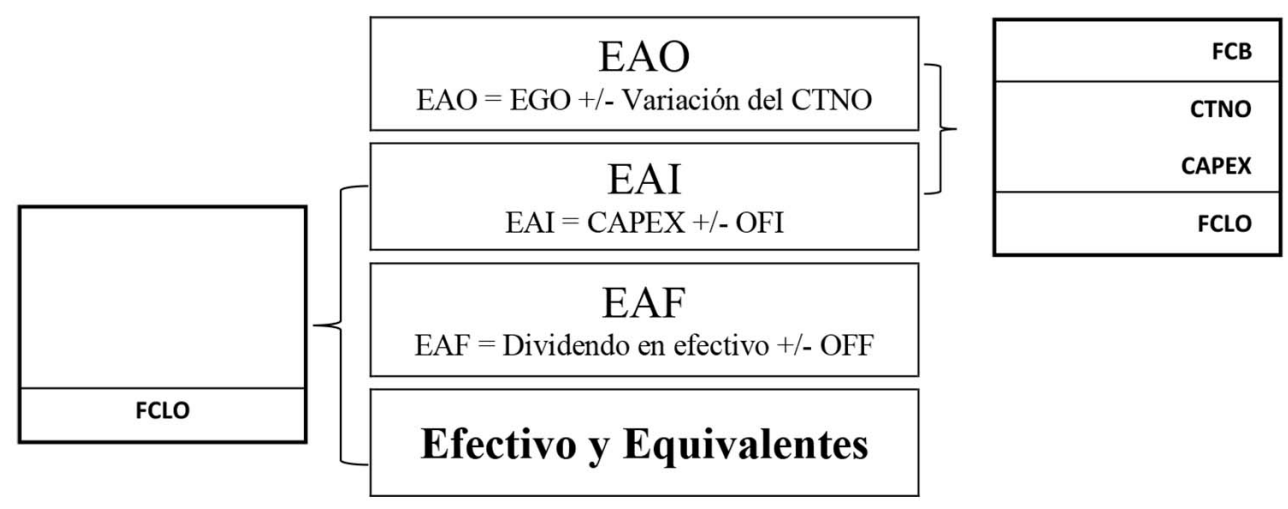

FIGURA 3

Relación entre EFE bajo NIC 7 y el Flujo de Caja Libre

Fuente: elaboración propia. 
Con la elaboración del EFE se logra una estructura cercana al FCL ya que basta con realizar algunas reclasificaciones especialmente en el flujo de inversión para identificar las partidas operativas y las no operativas, ya que las primeras se consideran en el CAPEX y hacen parte del Flujo de Caja Libre Operativo (FCLO) y los otros flujos de inversión hacen parte del flujo de caja libre financiero.

Con base en los fundamentos normativos y gerenciales en torno a los flujos de efectivo, planteamos la siguiente hipótesis de investigación:

Ho: La presentación y análisis del EFE bajo NIIF constituye una herramienta significativamente útil para la gestión financiera sostenible.

\section{Desarrollo metodológico}

Esta investigación es desarrollada a través de un enfoque cuantitativo de tipo descriptivo y de alcance correlacional. En este apartado se explica la muestra, variables utilizadas y el modelo de análisis aplicado en el estudio.

\section{Muestra}

El desarrollo del estudio se centró en las empresas de Colombia que reportaron información financiera a la Superintendencia de Sociedades durante el año 2018. La base de datos inicial estaba conformada por 20.788 empresas. Luego de analizar las características de la información reportada, se encontró que algunas empresas no reportaron la información sobre el EFE o existían inconsistencias en la información. Finalmente, luego de depurar la muestra inicial se estudiaron 14.979 empresas pertenecientes a 9 sectores de la economía como se muestra en la tabla 2.

TABLA 2

Composición de la muestra por sectores

\begin{tabular}{lrc}
\hline \multicolumn{1}{c}{ Sector } & Cantidad & $\begin{array}{c}\text { Frecuencia } \\
\text { relativa }\end{array}$ \\
\hline Agropecuario & 940 & 0,06 \\
Comercial & 6.022 & 0,40 \\
Construcción & 1.632 & 0,11 \\
Financiero & 703 & 0,05 \\
Industrial & 2.816 & 0,19 \\
Servicios & 1.892 & 0,13 \\
Telecomunicaciones & 454 & 0,03 \\
Transporte & 243 & 0,02 \\
Turismo & 277 & 0,02 \\
\hline Total & $\mathbf{1 4 . 9 7 9}$ & $\mathbf{1 , 0 0}$
\end{tabular}

Fuente: elaboración propia con base en clasificación sectorial de la Superintendencia de Sociedades.

Los sectores más representativos dentro de la muestra estudiada son el comercial (40\%), industrial (19\%), servicios (13\%) y construcción (11\%). Esta situación se presenta debido a que la Superintendencia de Sociedades tiene a su cargo la inspección, vigilancia y control de sociedades mercantiles que no se encuentran dentro del alcance de las demás Superintendencias.

Dentro del reporte que publica la Superintendencia de Sociedades, se diferencian las empresas según el marco normativo contable que aplican. En la tabla 3 se presenta la composición de la muestra de acuerdo con esta clasificación. 
TABLA 3

Muestra de acuerdo con el estándar implementado de la empresa

\begin{tabular}{lcc}
\hline Estándar & Cantidad & $\begin{array}{c}\text { Frecuencia } \\
\text { relativa }\end{array}$ \\
\hline NIIF Plenas & 3.081 & 0,21 \\
\hline NIIF Pymes & 11.898 & 0,79 \\
\hline Total & $\mathbf{1 4 . 9 7 9}$ & $\mathbf{1 , 0 0}$ \\
\hline
\end{tabular}

Fuente: elaboración propia.

\section{Variables}

Para analizar los resultados del EFE con un enfoque de sostenibilidad financiera se consideraron los resultados del EAO, EAI y EAF y las variables que se explican a continuación.

Calidad del EFE. Un aspecto fundamental que dirigió el análisis de los resultados fue mediante la estructuración de ocho escenarios de acuerdo con Terreno et al. (2020) y que están en sintonía con el trabajo de Sayari \& Mugan (2013) para identificar los riesgos de continuidad empresarial y la sostenibilidad financiera con base en los resultados agregados de este estado financiero.

TABLA 4

Escenarios de análisis del EFE

\begin{tabular}{lcccccccc}
\hline \multicolumn{1}{c}{ Flujo de Efectivo } & $\mathbf{1}$ & $\mathbf{2}$ & $\mathbf{3}$ & $\mathbf{4}$ & $\mathbf{5}$ & $\mathbf{6}$ & $\mathbf{7}$ & $\mathbf{8}$ \\
\hline $\begin{array}{l}\text { Efectivo por Actividades de Operación } \\
\text { (EAO) }\end{array}$ & $(+)$ & $(+)$ & $(+)$ & $(+)$ & $(-)$ & $(-)$ & $(-)$ & $(-)$ \\
$\begin{array}{l}\text { Efectivo por Actividades de Inversión } \\
\text { (EAI) }\end{array}$ & $(+)$ & $(+)$ & $(-)$ & $(-)$ & $(-)$ & $(-)$ & $(+)$ & $(+)$ \\
$\begin{array}{l}\text { Efectivo por Actividades de Financiación } \\
\text { (EAF) }\end{array}$ & $(+)$ & $(-)$ & $(+)$ & $(-)$ & $(+)$ & $(-)$ & $(+)$ & $(-)$ \\
\hline
\end{tabular}

Fuente: elaboración propia.

Según lo expuesto por Terreno et al. (2020) el escenario 4 es considerado como el ideal para lograr la sostenibilidad financiera ya que la empresa obtiene flujos de efectivo suficientes a partir de la explotación de su objeto social que le permiten realizar inversiones y cumplir sus compromisos financieros con acreedores y accionistas. De otro lado, el escenario 7 es considerado un escenario de declive empresarial ya que la explotación del objeto social de la empresa no genera flujos de efectivo y por ende se debe recurrir a la venta de activos e incurrir en préstamos para sostener la operación. De acuerdo con Arimany, Moya \& Viladecans (2015) el escenario 4 es considerado una situación de madurez empresarial, en tanto el escenario 7 se puede considerar con un escenario de deterioro financiero.

Utilidad Neta (U. Neta). Esta variable se consideró debido a que el Código de Comercio Colombiano o Decreto 410 de 1971 establece esta medida como el referente para distribuir dividendos por parte de los socios de una empresa. No obstante, debido a que este nivel de utilidad es diferente a una medida de caja, según Yaari et al. (2016) existe la posibilidad de que empresas con utilidades no generen flujos de efectivo, lo cual afecta negativamente la sostenibilidad financiera.

Flujo de caja libre operacional (FCLO). A través de esta variable se pretende medir la capacidad de las empresas para generar flujos de efectivos a partir de su operación considerando la inversión operacional de largo plazo (CAPEX) y para cumplir con sus compromisos adquiridos con acreedores financieros y accionistas. La ecuación 1 muestra la forma de cálculo. 


$$
F C L O=E A O+\text { Capex }
$$

El resultado de esta variable incide directamente en la sostenibilidad financiera empresarial debido a que como lo afirma Foerster, Tsagarelis \& Wang (2017), las empresas sostenibles son aquellas que generan flujos de caja operacional positivos y suficientes para cubrir el servicio de la deuda y los dividendos.

Cobertura combinada $(C C)$. Esta variable hace referencia a la capacidad que tiene una empresa para pagar los dividendos y su servicio de la deuda (abono a capital e intereses) a partir de su FCLO. Según lo expuesto por Correa-Garcia (2007) cuando el FCLO es suficiente para cubrir estos dos rubros, se suplen las dos destinaciones que tiene este concepto y por ende existe una situación financiera sostenible. La ecuación 2 muestra la forma de medición de esta variable.

$$
C C=\frac{F C L O}{S D+\text { Dividendos }}
$$

El resultado de esta variable muestra el número de veces que el FCLO alcanza a cubrir el pago del servicio de la deuda y los dividendos de las compañías. Las firmas que cuentan con una cobertura combinada igual o superior a 1 tienen una situación financieramente sostenible. En caso contrario debería replantearse la decisión de dividendos y/o la decisión de financiación.

\section{Modelo de análisis}

El análisis de la información del EFE se desarrolló a través del método biplot desarrollado por Gabriel (1971). Este método permite analizar $\mathrm{n}$ individuos de acuerdo con $p$ características numéricas. Para el caso de este estudio los individuos están representados en las 14.979 empresas y las características numéricas por las cuatro variables descritas anteriormente, formando una matriz $X(n x p)$.

Una de las principales ventajas del método biplot para el análisis de la información según Ortas, Alvarez, Jaussaud et al. (2015), es que este método permite una representación visual de dos dimensiones que muestra la relación entre los individuos y las variables. A través de este tipo de gráficos se identifican las correlaciones entre los individuos y las correlaciones entre las variables con los individuos.

De acuerdo con Gonzales-Rojas, Conde-Arango, \& Ochoa-Muñoz (2021), el análisis por componentes principales es el método comúnmente utilizado para realizar procesos de análisis multivariados. Esta técnica de análisis de datos permite simplificar las dimensiones de los datos e identificar las principales correlaciones existentes entre los parámetros utilizados. Adicionalmente, según Cárdenas, Noguera, Galindo et al. (2006) las regresiones estimadas a través de componentes principales permiten corregir la inestabilidad que producen los problemas de multicolinealidad.

\section{Resultados y discusión}

Inicialmente se presenta un análisis descriptivo de los resultados de las empresas analizadas, seguido de un análisis correlacional a través de diferentes gráficos biplot generados a partir de las variables consideradas con el fin de realizar un análisis multidimensional. La tabla 5 muestra la distribución en los 8 escenarios descritos de los resultados de los flujos de efectivo de las empresas analizadas. Se logra observar que el escenario 4 es el de 
mayor frecuencia, lo cual implica que el 25\% de las empresas analizadas se encuentran en una situación ideal desde la generación y aplicación de los flujos de efectivo, lo cual aporta para lograr la sostenibilidad financiera.

TABLA 5

Escenarios del flujo de efectivo

\begin{tabular}{ccc}
\hline E_EFE & Cantidad & Frecuencia \\
\hline 1 & 1.800 & 0,12 \\
2 & 1.676 & 0,11 \\
3 & 2.974 & 0,20 \\
4 & 3.713 & 0,25 \\
5 & 2.129 & 0,14 \\
6 & 271 & 0,02 \\
7 & 1.792 & 0,12 \\
8 & 624 & 0,04 \\
\hline Total & $\mathbf{1 4 . 9 7 9}$ & $\mathbf{1 , 0 0}$ \\
\hline
\end{tabular}

Fuente: elaboración propia.

El segundo escenario más común para las empresas analizadas es el 3, el cual es considerado de expansión empresarial (Terreno et al., 2020), ya que las inversiones requeridas son mayores a los flujos de efectivo logrados por la explotación del objeto social y por ende es necesario apalancar las inversiones con deudas con terceros o inversionistas (Yaari et al., 2016). A pesar de que el escenario 4 es el que más se repite dentro de las empresas analizadas, es importante resaltar que 4.816 empresas que representan el $32 \%$ de la muestra analizada cuentan con EAO negativos (escenarios 5 al 8), lo cual trae consecuencias negativas para la sostenibilidad financiera y el valor de las empresas ya que no se están generando flujos de efectivo a partir de la explotación del objeto social y por ende se debe recurrir a financiaciones externas para sostener financieramente el desarrollo del objeto social.

De acuerdo con los resultados logrados por Giriati (2016), no basta con que las empresas generen EAO positivos. Para que los resultados financieros sean sostenibles, los flujos de caja operacionales deben alcanzar a cubrir tanto las obligaciones con terceros como con accionistas (Yeo, 2018). Sin embargo, la legislación comercial (Decreto 410 de 1971) establece la utilidad neta como el valor de referencia para la decisión de dividendos, lo cual puede generar adversidades financieras en la medida en que la utilidad no es una medida de flujo de efectivo y las empresas podrían generar obligaciones efectivas con sus socios sin contar con la capacidad de cubrirlas (Yaari et al., 2016). En la figura 4 se analizan los resultados de la utilidad neta, el EAO y el FCLO como medidas referentes para las decisiones de endeudamiento y dividendos empresariales. 


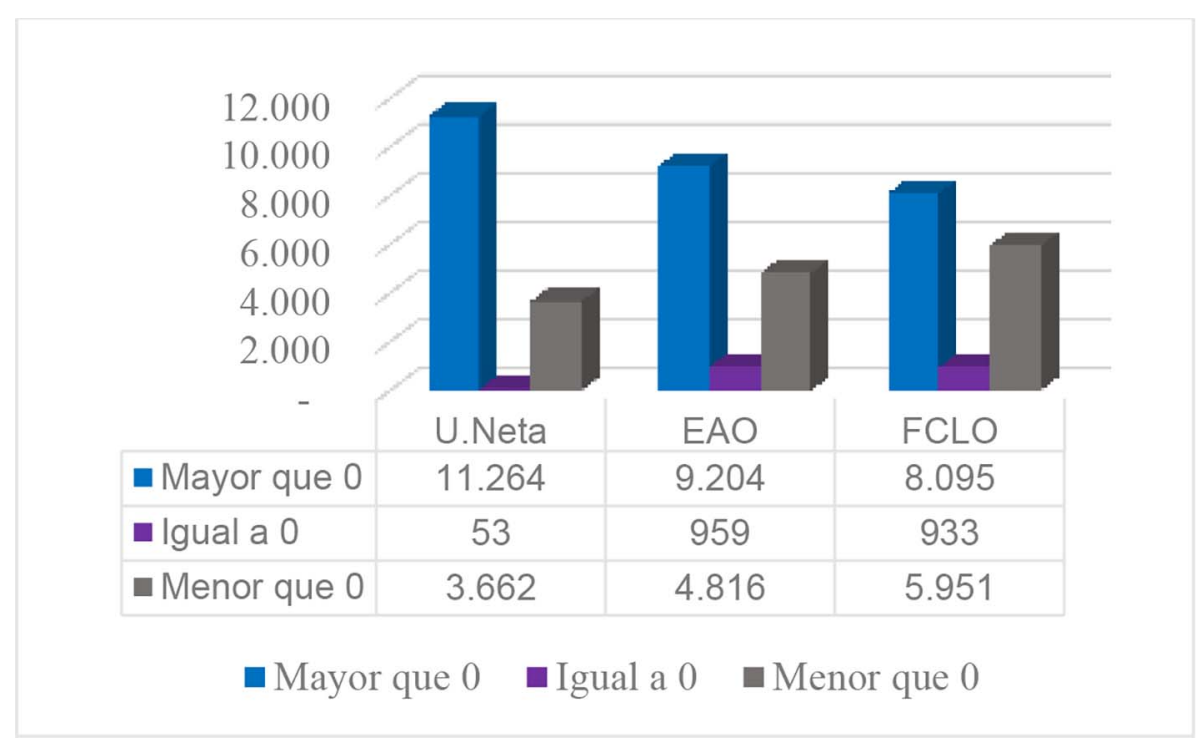

FIGURA 4

Medidas de capacidad de pago

Fuente: elaboración propia.

A partir de la figura 4 se evidencia que hay una mayor cantidad de empresas que tienen utilidad neta positiva que el EAO y el FCLO, lo que puede llevar a las empresas a distorsionar las decisiones de financiación y de dividendos empresariales. En la tabla 6 se presenta la cantidad de empresas que cuentan con capacidad para cubrir a partir de sus FCLO sus obligaciones con acreedores financieros (servicio de la deuda) y accionistas.

TABLA 6

Cobertura combinada

\begin{tabular}{ccc}
\hline Escenario & Cantidad & Frecuencia \\
\hline Mayor o igual que 1 & 6.552 & 0,44 \\
\hline Menor que 1 & 8.427 & 0,56 \\
\hline Total & $\mathbf{1 4 . 9 7 9}$ & $\mathbf{1 , 0 0}$ \\
\hline
\end{tabular}

Fuente: elaboración propia.

Para las empresas analizadas, se evidencia que la mayor parte (56\%) no alcanza a cubrir sus obligaciones con terceros y accionistas a partir de los flujos de caja que generan en sus operaciones. Esta situación afecta negativamente la sostenibilidad financiera empresarial en la medida que estas empresas deberán adquirir nuevas deudas con acreedores financieros y/o accionistas o realizar desinversiones para atender sus compromisos. Adicional a que el FCLO alcance a cubrir el servicio de la deuda y los dividendos, se debe considerar que para que esta situación financiera sea sostenible financieramente debe estar sustentado en EAO positivos. Este análisis se puede advertir en la tabla 7. 
TABLA 7

Cobertura combinada y escenarios EFE

\begin{tabular}{crrr}
\hline E_EFE $^{*}$ & Mayor que 1 & Menor que 1 & Total \\
\hline 1 & 831 & 969 & 1.800 \\
2 & 1.223 & 453 & 1.676 \\
3 & 1.721 & 1.253 & 2.974 \\
4 & 2.579 & 1.134 & 3.713 \\
5 & 5 & 2.124 & 2.129 \\
6 & 1 & 270 & 271 \\
7 & 106 & 1.686 & 1.792 \\
8 & 86 & 538 & 624 \\
\hline Total & $\mathbf{6 . 5 5 2}$ & $\mathbf{8 . 4 2 7}$ & $\mathbf{1 4 . 9 7 9}$ \\
\hline${ }^{*}$ Escenario del EFE & & &
\end{tabular}

Fuente: elaboración propia.

A través de la tabla 7 se aprecia que hay empresas con indicador de cobertura combinada mayor que 1 a pesar de contar con EAO negativos (escenarios del 5 al 8). Esta situación se presenta debido a la desinversión en activos operacionales de largo plazo (ejemplo: propiedad, planta y equipo), lo cual según Park \& Jang (2013) significaría una pérdida de capacidad de las empresas para operar en el futuro. Igualmente, se observa que las empresas que se encuentran en el escenario 4 del EFE son más proclives a contar con capacidad de cobertura combinada, con lo cual se ratifica que es el escenario ideal para la gestión financiera sostenible.

La tabla 8 muestra las estadísticas descriptivas de las variables consideradas en este estudio. El resumen estadístico muestra los resultados de la muestra total, las empresas que tienen una cobertura combinada mayor que uno y menor que uno, así como los resultados de las empresas que se encuentran dentro del escenario 4 y 7 del EFE. Los resultados muestran que en general existe una distancia importante entre la utilidad neta, el EAO y el FCLO, siendo este último menor. Las estadísticas descriptivas de las empresas con cobertura combinada menor que 1 ratifican la posibilidad de que existan empresas con utilidad neta favorable pero que no generan flujos de efectivos operacionales (EAO ni FCLO) para afrontar sus obligaciones con acreedores y accionistas.

TABLA 8

Estadísticas descriptivas

\begin{tabular}{|c|c|c|c|c|c|c|c|}
\hline & & Media & Mediana & Desviación est. & Mínimo & Máximo & Observaciones \\
\hline \multirow{5}{*}{$\begin{array}{l}\text { Muestra } \\
\text { total }\end{array}$} & U. Neta & 3.059 .904 & 284.448 & 55.256 .273 & -802.449 .716 & 3.575 .752 .687 & 14.979 \\
\hline & EAO & 3.695 .156 & 215.848 & 75.621 .795 & $\begin{array}{l}-1.965 .380 .635 \\
\end{array}$ & 4.729 .036 .701 & 14.979 \\
\hline & EAI & -2.168 .214 & $\begin{array}{l}-42.388 \\
\end{array}$ & 49.733 .957 & -3.433 .066 .630 & 2.388 .637 .055 & 14.979 \\
\hline & $\begin{array}{l}\text { EAI } \\
\text { EAF }\end{array}$ & $\begin{array}{l}-2.1681 .214 \\
-1.321 .925\end{array}$ & -42.588 & $\begin{array}{l}49.1310 .931 \\
57.710328\end{array}$ & $\begin{array}{l}-3.435 .000 .030 \\
-4.961 .355 .000\end{array}$ & $\begin{array}{l}2.388 .637 .053 \\
1.032 .758 .006\end{array}$ & $\begin{array}{l}14.979 \\
14.979\end{array}$ \\
\hline & FCLO & 1.873 .723 & 38.751 & 56.352 .437 & -752.521 .085 & 4.302 .331 .701 & 14.979 \\
\hline \multirow{5}{*}{$\mathrm{CC}>1$} & U. Neta & 5.986 .299 & 463.106 & $\begin{array}{l}50.532 .451 \\
78.650 .471\end{array}$ & -119.341 .000 & $\begin{array}{l}4.502 .531 .101 \\
.575 .752 .687\end{array}$ & $\frac{14.919}{6.552}$ \\
\hline & EAO & 9.008 .737 & 1.051 .537 & 102.478 .378 & -1.747 .782 .927 & 4.729 .036 .701 & 6.552 \\
\hline & EAI & -2.593 .783 & -70.200 & 52.605 .810 & -2.475 .004 .424 & 2.388 .637 .055 & 6.552 \\
\hline & EAF & -5.313 .565 & -129.314 & 83.094.576 & -4.961 .355 .000 & 291.787 .382 & 6.552 \\
\hline & FCLO & 7.418 .439 & 851.763 & 82.611 .244 & 1 & 4.302 .331 .701 & 6.552 \\
\hline \multirow{5}{*}{$\mathrm{CC}<1$} & U. Neta & 784.629 & 182.789 & 24.621 .801 & -802.449 .716 & 880.867 .184 & 8.427 \\
\hline & EAO & -436.158 & -31.123 & 44.289 .092 & -1.965 .380 .635 & 3.247 .838 .041 & 8.427 \\
\hline & EAI & $\begin{array}{r}-1.837 .335 \\
\end{array}$ & -22.513 & 47.381.497 & -3.433 .066 .630 & 1.964 .553 .863 & 8.427 \\
\hline & EAF & 1.781 .580 & & 23.021 .399 & -292.188 .122 & 1.032 .758 .006 & 8.427 \\
\hline & FCLO & $\begin{array}{r}1.437 .298 \\
\end{array}$ & -235.826 & 17.218 .027 & -752.521 .085 & 357.347 .098 & 8.427 \\
\hline \multirow{5}{*}{ E_EFE 4} & U. Neta & 6.637 .081 & 632.277 & 81.344 .358 & -180.622 .342 & 3.575 .752 .687 & 3.713 \\
\hline & EAO & 14.636 .265 & 1.705 .794 & 137.082 .410 & 1 & 4.729 .036 .701 & 3.713 \\
\hline & EAI & $\begin{array}{r}14.030 .203 \\
-6.052 .603\end{array}$ & $\begin{array}{l}1.7597 .208 \\
-397\end{array}$ & 79.014 .304 & $\begin{array}{r}-3.433 .066 .630 \\
\end{array}$ & $\begin{array}{r}4.129 .030 .101 \\
-2\end{array}$ & 3.713 \\
\hline & EAF & -8.264 .347 & -813.688 & 101.890 .828 & -4.961 .355 .000 & -33 & 3.713 \\
\hline & FCLO & 9.362 .600 & 1.116 .864 & 101.070 .018 & $\begin{array}{r}-185.236 .572 \\
\end{array}$ & 4.302 .331 .701 & 3.713 \\
\hline \multirow{5}{*}{ E_EFE 7} & U. Neta & -89.544 & 44.698 & 23.582 .117 & -470.549 .070 & 572.568 .196 & 1.792 \\
\hline & EAO & -5.105 .717 & -536.485 & 48.658 .775 & -1.965 .380 .635 & -2 & 1.792 \\
\hline & EAI & 2.458 .288 & 15.921 & 46.893 .309 & - & 1.964 .553 .863 & 1.792 \\
\hline & EAF & $\begin{array}{l}2.4357 .290 \\
2.359\end{array}$ & & 12.193 .207 & & 249.047 .217 & 1.792 \\
\hline & FCLO & $\begin{array}{r}-3.628 .771 \\
\end{array}$ & -353.088 & 15.042 .472 & -231.120 .877 & 42.739 .570 & 1.792 \\
\hline
\end{tabular}

Fuente: elaboración propia.

Para agrupar las empresas de acuerdo con las UN, EAO, EAI, EAF y FCLO, se implementó la técnica del biplot para todas las compañías. Este análisis se realizó para la muestra total, para las empresas con cobertura combinada mayor y menor que 1 y para las empresas que se encuentran en el escenario 4 y 7 del EFE. En la tabla 9 se muestra la importancia de los componentes principales (PC) en cada agrupación de datos. 
TABLA 9

Importancia de los componentes principales

\begin{tabular}{|c|c|c|c|c|c|}
\hline \multicolumn{6}{|c|}{ Muestra total } \\
\hline & PC1 & PC2 & PC3 & PC4 & PC5 \\
\hline Desviación estándar & 1,3995 & 1,0718 & 0,9989 & 0,9200 & 0,22007 \\
\hline Proporción de varianza & 0,3917 & 0,2297 & 0,1996 & 0,1693 & 0,00969 \\
\hline Proporción acumulada & 0,3917 & 0,6215 & 0,8210 & 0,9903 & 1 \\
\hline \multicolumn{6}{|c|}{$\mathrm{CC}>1$} \\
\hline & PC1 & PC2 & PC3 & PC4 & PC5 \\
\hline Desviación estándar & 1,4795 & 1,0114 & 0,9755 & 0,9124 & 0,06355 \\
\hline Proporción de varianza & 0,4378 & 0,2046 & 0,1903 & 0,1665 & 0,00081 \\
\hline Proporción acumulada & 0,4378 & 0,6424 & 0,8327 & 0,9992 & 1 \\
\hline \multicolumn{6}{|c|}{$\mathrm{CC}<1$} \\
\hline & PC1 & PC2 & $\mathbf{P C 3}$ & PC4 & PC5 \\
\hline Desviación estándar & 1,4264 & 1,1458 & 0,9835 & 0,60901 & 0,56063 \\
\hline Proporción de varianza & 0,4069 & 0,2626 & 0,1935 & 0,07418 & 0,06286 \\
\hline Proporción acumulada & 0,4069 & 0,6695 & 0,863 & 0,93714 & 1 \\
\hline \multicolumn{6}{|c|}{ E_EFE 4} \\
\hline & PC1 & PC2 & PC3 & PC4 & PC5 \\
\hline Desviación estándar & 1,6461 & 1,0162 & 0,9037 & 0,63460 & 0,19595 \\
\hline Proporción de varianza & 0,5419 & 0,2065 & 0,1633 & 0,08054 & 0,00768 \\
\hline Proporción acumulada & 0,5419 & 0,7484 & 0,9118 & 0,99232 & 1 \\
\hline \multicolumn{6}{|c|}{ E_EFE 7} \\
\hline & PC1 & PC2 & PC3 & PC4 & PC5 \\
\hline Desviación estándar & 1,4099 & 1,0534 & 1,0025 & 0,9327 & 0,1658 \\
\hline Proporción de varianza & 0,3976 & 0,2219 & 0,2010 & 0,1740 & 0,0055 \\
\hline Proporción acumulada & 0,3976 & 0,6195 & 0,8205 & 0,9945 & 1 \\
\hline
\end{tabular}

CC: Cobertura combinada; E_EFE: Escenario del EFE; PC: Componente principal.

Fuente: elaboración propia.

En todos los casos la varianza es explicada en más del $60 \%$ por los dos primeros componentes principales y en el tercer componente se llega a un grado de explicación superior al 80\%. Por tanto, al contar con una mayor explicación en los dos primeros componentes principales, estos fueron los analizados en las representaciones gráficas biplot. Inicialmente, se presenta la representación biplot considerando las 14.979 empresas que componen la muestra del estudio realizado y agrupándolas de acuerdo con su cobertura combinada, ver figura 5.

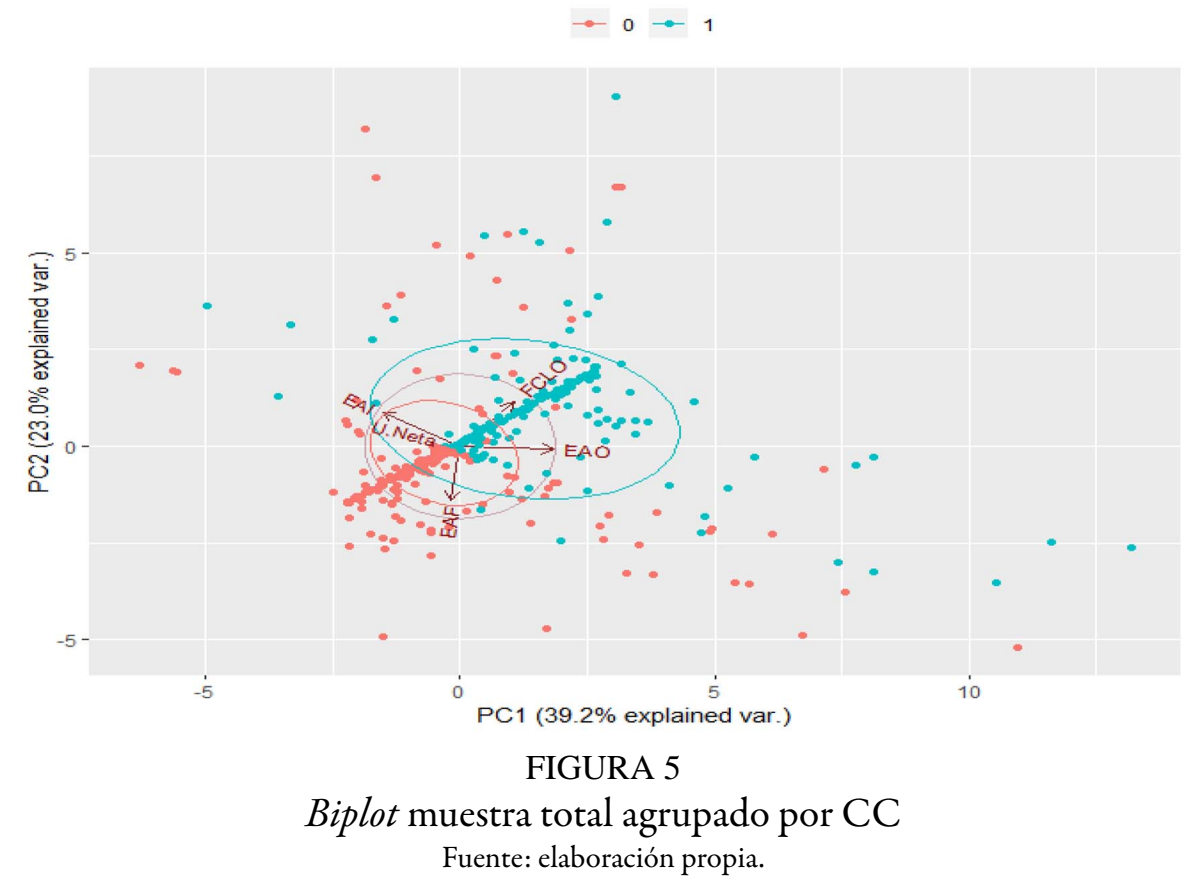


Algunas empresas fueron omitidas de la figura con el fin de aumentar la legibilidad. Para analizar los resultados de la figura 5 se considera la extensión y dirección de cada vector (variable) con respecto al componente principal 1 (eje x) y 2 (eje y). Adicionalmente, el sentido y la distancia entre cada variable representa el nivel y el sentido de correlación. La muestra total fue agrupada en dos conjuntos. El primero corresponde a las empresas que no cuentan con capacidad de cobertura del servicio de la deuda y dividendos (color rojo) y el segundo corresponde a las empresas que cuentan con capacidad para cubrir estas obligaciones (color azul).

A partir de la figura 5 se evidencia que en términos generales existe una correlación positiva entre el FCLO y el EAO, sin embargo, esta correlación no es igual para la utilidad neta. Lo anterior indica que las empresas que generan utilidades netas positivas no necesariamente cuentan con flujos de caja que les permita atender sus acreencias financieras y dividendos, lo cual es coherente con lo encontrado en Correa-Mejía \& Lopera-Castaño (2019). Adicionalmente, la distancia entre el EAI y el EAF con el FCLO y el EAO indica la existencia de una correlación negativa entre estas variables. Lo anterior es coherente con lo hallado por Dewi, Sari, Budiasih et al. (2019) ya que la generación de flujos de caja operativos genera la posibilidad de realizar inversiones y pagar obligaciones con terceros y accionistas.

El análisis de los subgrupos desagregados de la muestra total permite confirmar que las empresas con CC mayor que 1 se concentran cerca de las variables del FCLO y EAO, lo que muestra la capacidad de estas empresas para generar flujos de efectivo a partir de sus operaciones. Este resultado va en vía con lo encontrado por Park \& Jang, (2013). Por su parte, las empresas con CC menor que 1 generan sus flujos de efectivo a partir de las actividades de inversión y financiación. La figura 6 presenta el biplot agrupado por los escenarios 4 y 7 del EFE.

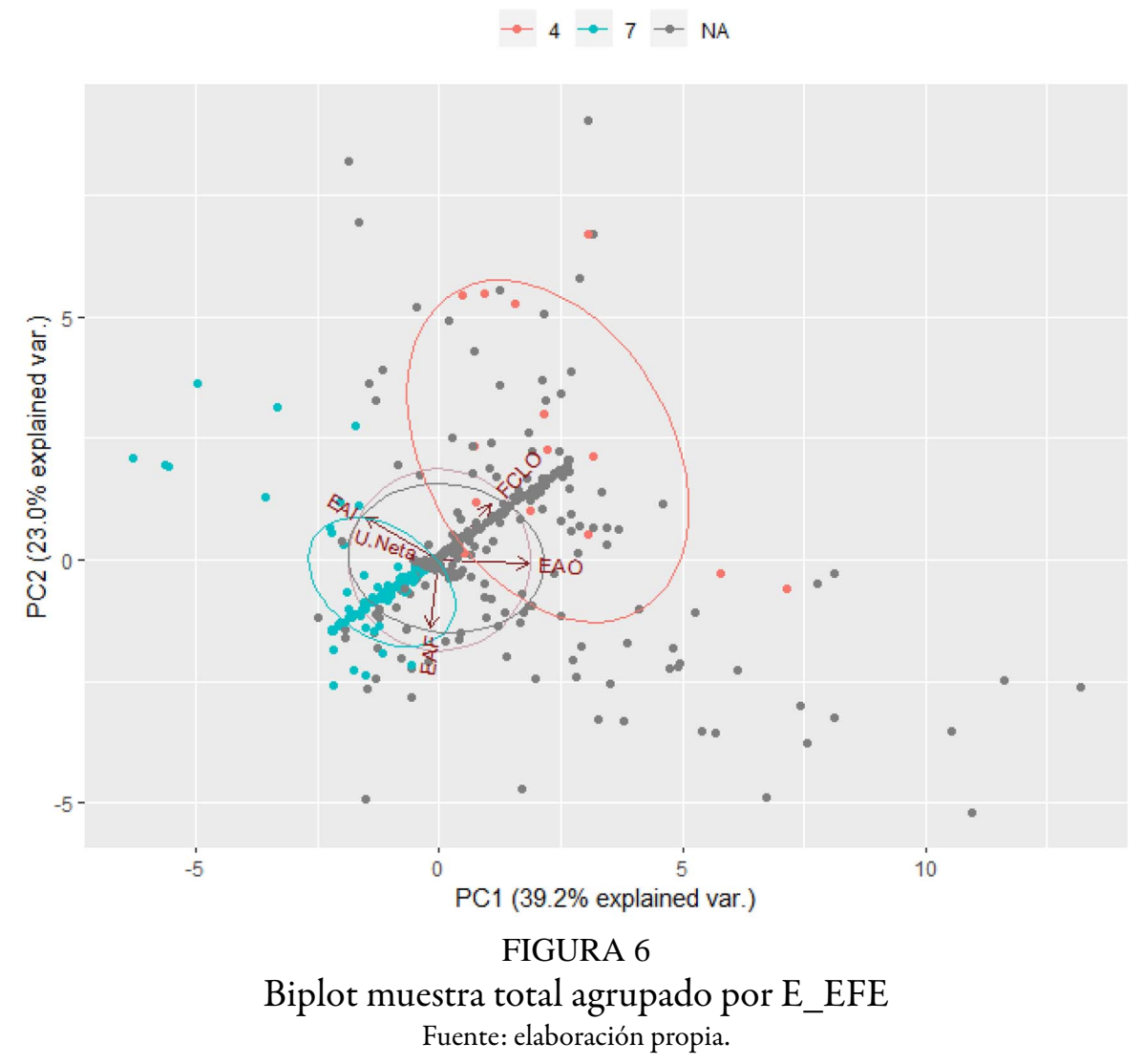

La figura 6 muestra que las empresas que se encuentran en el escenario 4 poseen una concentración hacia los FCLO positivos y gran parte del área compuesta por las empresas de este escenario es compartida con las empresas que cuentan con una CC mayor que 1 , lo que indica que las empresas que cuentan con un escenario 
ideal en sus EFE tienen mayor probabilidad de tener la capacidad de pago de obligaciones con acreedores financieros y accionistas. El caso opuesto sucede con las empresas que pertenecen al escenario 7 del EFE, donde comparten gran parte de su área con las empresas que cuentan con una CC menor que 1. Las empresas clasificadas en el grupo NA son las que se encuentran en los demás escenarios del EFE que no son considerados en este análisis.

Las herramientas y técnicas de análisis aplicadas en este estudio para el EFE y en su estrecha vinculación con el FCL, permiten afirmar la hipótesis de este artículo de investigación en el sentido de establecer que el EFE presentado de conformidad con las NIIF constituye una herramienta significativamente útil para la gestión financiera sostenible en las empresas. Esta validación a partir de le evidencia empírica de 14.979 empresas no cotizadas está en línea con Kolmakov \& Polyakova (2019), Charitou et al. (2018), Kajananthan \& Velnampy, (2014) que plantean las bondades y potencialidades de los flujos de caja en la valoración y gestión empresarial.

\section{Conclusiones}

En este trabajo se presentaron ocho escenarios de análisis para el EFE. Igualmente se evidenciaron las conexiones que existen entre el EFE y otros flujos de caja no regulados, pero de alta aplicación en finanzas y de amplio interés académico a partir de la teoría de la agencia como lo es el Flujo de Caja Libre (Shyam \& Mollie, 2017). Los resultados mostraron que cerca del 32\% de las empresas colombianas presentan flujos operacionales negativos. Este comportamiento de mantenerse en el largo plazo constituye una señal de alerta para la gestión financiera sostenible, puesto que implicaría dificultades para la realización de inversiones, atender compromisos financieros y pagar dividendos.

El análisis de escenarios realizado para las empresas colombianas con base en Terreno et al. (2020) evidenció que el escenario 4 es el de mayor frecuencia con un $25 \%$ de las compañías registrando una situación ideal en el comportamiento de sus flujos de efectivo lo cual es una condición favorable para la gestión financiera sostenible. El segundo escenario con mayor representatividad fue el escenario 3 considerado de expansión empresarial, lo que denota igualmente que las empresas colombianas en este período contaban con perspectivas de crecimiento. De otro lado, se encontró que el 32\% de las empresas analizadas contaban con flujos operacionales negativos (escenarios 5 al 8) lo cual es una señal de alerta para la continuidad y sostenibilidad empresarial. Estos resultados y los análisis de indicadores realizados para estos escenarios evidencian la importancia de utilizar el EFE como herramienta constante de las finanzas empresariales.

Estos resultados, amén del detalle de análisis logrados con la generosa base de datos de la Superintendencia de Sociedades, tiene implicaciones relevantes tanto para los preparadores de la información contable como para los distintos usuarios, especialmente inversores y analistas, ya que evidencian cómo a partir del EFE se pueden estructurar análisis que apoyen la gestión financiera de largo plazo. La estructuración y estudio en detalle del flujo de caja libre y los indicadores que posibilita este estado financiero, constituyen otra herramienta importante que puede ser utilizada por las empresas, profesionales y entidades de vigilancia y control para advertir desde una perspectiva más financiera y de gestión del valor, los riesgos de continuidad que pueden enfrentar las empresas colombianas (Kamaluddin, Ishak, \& Mohammed, 2019). La aplicación del análisis biplot para analizar multidimensionalmente las correlaciones entre las variables del EFE de diferentes grupos muestrales también constituye un aporte de esta investigación, toda vez que, hasta lo mejor de nuestro conocimiento, durante la revisión teórica del estudio no se hallaron estudios similares para el flujo de caja bajo NIIF siguiendo esta metodología.

Este estudio presenta dos limitaciones principalmente. En primer lugar, se ocupa de un solo país emergente como lo es Colombia y, en segundo lugar, el estudio de corte transversal analiza la información correspondiente sólo al año 2018. Sin embargo, Colombia presenta una base de datos relevante de empresas no cotizadas que posibilita la realización de estudios de esta naturaleza y aunque los resultados no puedan ser 
extrapolados a otros contextos, la propuesta metodológica aplicada en este estudio puede ser replicada para otras economías emergentes. De otro lado, el considerar sólo el año 2018 se sustenta en la reciente la aplicación de las NIIF en Colombia y que los cambios presentados en el reporte de información a las Superintendencia de Sociedades llevan a que la información sobre el EFE aún esté en proceso de maduración y ajuste.

Con los resultados de este estudio se sientan las bases para trabajos posteriores que exploren los factores determinantes de los resultados que muestra el EFE, el FCL y por tanto cómo adoptar medidas que contribuyan con una gestión financiera sostenible que en un enfoque de largo plazo apoye la creación de valor empresarial. Asimismo se abre la posibilidad a la realización de trabajos comparativos en el contexto latinoamericano y global. De esta manera contribuimos a la literatura que establece las relaciones entre la contabilidad y las finanzas en un entorno preocupado por la sostenibilidad empresarial, Asimismo aportamos en estudios sobre este estado financiero que ha sido de baja utilización en la cultura contable colombiana.

\section{Consideraciones éticas}

Este estudio no requirió de aval ético ya que se fundamenta principalmente en datos de naturaleza pública.

\section{Contribución de los autores}

Los autores contribuyeron en iguales proporciones en la planificación, desarrollo y análisis de los resultados de esta investigación.

\section{Financiación}

Este trabajo es un resultado del proyecto de investigación "El Reporte Corporativo en Colombia y América Latina como mecanismo de rendición de cuentas" financiado por la Universidad de Antioquia. Acta de inicio: 2019-26130

\section{Conflictos de interés}

Los autores declaran no tener ningún conflicto de intereses.

\section{Referencias}

Agudelo-Rodríguez, L., Flórez-Londoño, A., \& Correa-Mejía, D. (2020). Efectos de la aplicación del marco normativo para entidades de gobierno en los indicadores financieros. Entramado, 16(2), 90-110. https://doi.org/10.1804 $1 / 1900-3803 /$ entramado.2.6545

Arimany, N., Moya, S., \& Viladecans, C. (2015). Utilidad del Estado de Flujos de Efectivo para el análisis empresarial. Revista de Contabilidad Y Dirección, 20, 195-217. https://dialnet.unirioja.es/servlet/articulo?codigo=5250483

Baik, B., Cho, H., Choi, W., \& Lee, K. (2016). Who classifies interest payments as financing activities? An analysis of classification shifting in the statement of cash flows at the adoption of IFRS. Journal of Accounting and Public Policy, 35(4), 331-351. https://doi.org/10.1016/j.jaccpubpol.2015.11.003

Baños-Caballero, S., García-Teruel, P., \& Martínez-Solano, P. (2014). Working capital management, corporate performance, and financial constraints. Journal of Business Research, 67(3), 332-338. https://doi.org/10.1016 /j.jbusres.2013.01.016 
Bendickson, J., Muldoon, J., Liguori, E., \& Davis, P. (2016). Agency theory: Background and epistemology. Journal of Management History 22(4),437-449 https://doi.org/10.1108/JMH-06-2016-0028

Bradbury, M. (2011). Direct or Indirect Cash Flow Statements? Australian Accounting Review, 21(57), 124-130. ht tps://doi.org/10.1111/j.1835-2561.2011.00130.x

Cárdenas, O., Noguera, C., Galindo, P., \& Vicente-Villardón, J. (2006). Alternativa a la regresión con componentes principales basada en biplot de regresión. Interciencia, 31(3), 160-167. http://ve.scielo.org/scielo.php?script=s ci_arttext\&pid=S0378-18442006000300004

Charitou, A., Karamanou, I., \& Kopita, A. (2018). The determinants and valuation effects of classification choice on the statement of cash flows. Accounting and Business Research, 48(6), 613-650. https://doi.org/10.1080/0001 4788.2017.1407626

Chen, A., \& Gong, J. (2019). Accounting comparability, financial reporting quality, and the pricing of accruals. Advances in Accounting, 45, 100415. https://doi.org/10.1016/j.adiac.2019.03.003

Correa-Garcia, J. (2007). Análisis comparativo: el Ebitda y el flujo de caja libre -FCL- y su aplicación en la gestión financiera empresarial. Revista Internacional Legis de Contabilidad \& Auditoria, 1(32), 159-181. https://www .legis.com.co/revista-internacional-contabilidad-auditoria/p

Correa, D., Martínez, L., Ruiz, M., \& Yepes, M. (2018). Los indicadores de costos: una herramienta para gestionar la generación de valor en las empresas industriales colombianas. Estudios Gerenciales, 34(147), 190-199. https:// doi.org/10.18046/j.estger.2018.147.2643

Correa, J. (2005). De la partida doble al análisis financiero. Contaduria Universidad de Antioquia, (46), 169-194. ht tps://revistas.udea.edu.co/index.php/cont/article/view/25682

Correa, J., Pulgarín, A., Muñoz, L., \& Álvarez, M. (2010). Marco normativo y antecedentes de la revelación contable en Colombia. Contaduria Universidad de Antioquia, (56), 269-292. https://revistas.udea.edu.co/index.php/c ont/article/view/14703

Correa-Mejía, D., \& Lopera-Castaño, M. (2019). Pronóstico de insolvencia empresarial en Colombia a través de indicadores financieros. Panorama Económico, 27, 510-526. https://doi.org/10.32997/2463-0470-vol.27-num .2-2019-2639

Correa-Mejía, D., \& Lopera-Castaño, M. (2020). Financial ratios as a powerful instrument to predict insolvency; a study using boosting. Estudios gerenciales, 36(155), 229-238. https://doi.org/10.18046/j.estger.2020.155.3588

Dewi, I., Sari, M., Budiasih, I., \& Suprasto, H. (2019). Free Cash Flow Effect towards Firm Value. International Research Journal of Management, IT y Social Sciences, 6(3), 108-116. https://doi.org/https://doi.org/10.21744/irjmis. v6n3.643

Duque, A. (2015). Estado de flujos de efectivo: aplicación de razonamientos algebraicos y de la NIC 7. Cuadernos de Contabilidad, 16(40), 73-109. https://doi.org/10.11144/Javeriana.cc16-40.efea

Estridge, J., \& Lougee, B. (2007). Measuring free cash flows for equity valuation: Pitfalls and possible solutions. Journal of Applied Corporate Finance, 19(2), 60-71. https://doi.org/10.1111/j.1745-6622.2007.00136.x

Foerster, S., Tsagarelis, J., \& Wang, G. (2017). Are Cash Flows Better Stock Return Predictors Than Profits? Financial Analysts Journal, 73(1), 73-99. https://doi.org/10.2139/ssrn.2472571

Gabriel, K. (1971). The biplot graphic display of matrices with application to principal component analysis, Biometrika, 58(3), 453-467. https://doi.org/10.1093/biomet/58.3.453

Giriati. (2016). Free Cash Flow, Dividend Policy, Investment Opportunity Set, Opportunistic Behavior and Firm's Value: (A Study About Agency Theory). Procedia Social and Behavioral Sciences, 219, 248-254. https://doi.or $\mathrm{g} / 10.1016 / \mathrm{j}$. sbspro.2016.05.013

Gonzales-Rojas, V., Conde-Arango, G., \& Ochoa-Muñoz, A. (2021). Análisis de Componentes Principales en presencia de datos faltantes: el principio de datos disponibles. Scientia et Technica, 26(2), 219-228. https://doi .org/10.22517/23447214.20591

Gordon, E., Henry, E., Jorgensen, B., \& Linthicum, C. (2017). Flexibility in cash-flow classification under IFRS: determinants and consequences. Review of Accounting Studies, 22(2), 839-872. https://doi.org/10.1007/s111 42-017-9387-1 
Hales, J., \& Orpurt, S. (2013). A Review of Academic Research on the Reporting of Cash Flows from Operations. Accounting Horizons, 27(3), 539-578. https://doi.org/10.2308/acch-50498

Jensen, M. (1986). Agency Costs of Free Cash Flow, Corporate Finance, and Takeovers. American Economic Review, 76(2), 323-329. https://www.jstor.org/stable/1818789?seq=1\#page_scan_tab_contents

Kajananthan, R., \& Velnampy, T. (2014). Liquidity, Solvency and Profitability Analysis Using Cash Flow Ratios and Traditional Ratios\#: The Telecommunication Sector in Sri Lanka. Research Journal of Finance and Accounting, 5(23), 163-171. https://www.iiste.org/Journals/index.php/RJFA/article/view/18769

Kamaluddin, A., Ishak, N., \& Mohammed, N. (2019). Financial Distress Prediction Through Cash Flow Ratios Analysis. International Journal of Financial Research, 10(3), 63. https://doi.org/10.5430/ijfr.v10n3p63

Kroes, J., \& Manikas, A. (2014). Cash flow management and manufacturing firm financial performance: A longitudinal perspective. International Journal of Production Economics, 148, 37-50. https://doi.org/10.1016/j.ijpe.2013.1 1.008

Kolmakov, V., \& Polyakova, A. (2019). Regional free cash flow dataset: An approach to regional performance evaluation. Data in Brief, 25, 1-5. https://doi.org/10.1016/j.dib.2019.104175

Kousenidis, D. (2006). A free cash flow version of the cash flow statement: a note. Managerial Finance, 32(8), 645653. https://doi.org/10.1108/03074350610676741

Mascareñas, J. (2013). Introducción a las Finanzas Corporativas. Monografías sobre Finanzas Corporativas. https:// ssrn.com/abstract $=2313264$

Orpurt, S., \& Zang, Y. (2007). Do Direct Cash Flow Disclosures Help Predict Future Operating Cash Flows and Earnings? The Accounting Review, 84(3), 893-935. https://doi.org/10.2308/accr.2009.84.3.893

Ortas, E., Alvarez, I., Jaussaud, J., \& Garayar, A. (2015). The impact of institutional and social context on corporate environmental, social and governance performance of companies committed to voluntary corporate social responsibility initiatives. Journal of Cleaner Production, 108, 673-684. https://doi.org/10.1016/j.jclepro.2015 .06 .089

Park, K., \& Jang, S. (2013). Capital structure, free cash flow, diversification and firm performance: A holistic analysis. International Journal of Hospitality Management, 33, 51-63. https://doi.org/10.1016/j.ijhm.2013.01.007

Rivera-Godoy, J., \& Rendón-Perea, J., (2019). Sector avícola en Colombia: rendimiento contable y EVA. Contaduría Universidad de Antioquia, 74, 127-151. Doi: https://doi.org/10.17533/udea.rc.n74a06

Sayari, N., \& Mugan, F. (2013). Cash Flow Statement as an Evidence for Financial Distress. Universal Journal of Accounting and Finance, 1(3), 95-103. https://doi.org/10.13189/ujaf.2013.010302

Shyam, B., \& Mollie, T. (2017). On the Definition, Measurement, and Use of the Free Cash Flow concept in financial reporting and analysis: A review and recommendations. Journal of Accounting and Finance, 17(1), 11-19. http s://doi.org/10.33423/jaf.v17i1.972

Sierra, E. (2001). Evolución de la normativa contable en Colombia. Revista Innovar, 17, 47-65. https://revistas.unal .edu.co/index.php/innovar/article/view/24230

Soboleva, Y., Matveev, V., Ilminskaya, S., Efimenko, I., Rezvyakova, I., \& Mazur, L. (2018). Monitoring of businesses operations with cash flow analysis. International Journal of Civil Engineering and Technology, 9(11), 2034-2044. http://ogiik.orel.ru/efimenko_is/Efimenko_Scopus1.pdf

Terreno, D., Peréz, J., \& Sattler, S. (2020). La relación entre liquidez, rentabilidad y solvencia: Una investigación empírica por el modelo de ecuaciones estructurales. Contaduria Universidad De Antioquia, (77), 13-35. https: //doi.org/10.17533/udea.rc.n77a01

Vishwanath, S., \& Krishnamurti, C. (2009). Investment Management: A Modern Guide to Security Analysis and Stock Selection. Nagpur, India: Springer-Verlag Berlin Heidelberg. https://doi:10.1007/978-3-540-88802-46

Yaari, U., Nikiforov, A., Kahya, E., \& Shachmurove, Y. (2016). Finance methodology of Free Cash Flow. Global Finance Journal, 29, 1-11. https://doi.org/10.1016/j.gfj.2015.05.003

Yeo, H. (2018). Role of free cash flows in making investment and dividend decisions: The case of the shipping industry. Asian Journal of Shipping and Logistics, 34(2), 113-118. https://doi.org/10.1016/j.ajsl.2018.06.007 
Jaime Andrés Correa-García a, et al. Importancia del estado de flujos de efectivo para la g...

Zhang, E. (2019). The impact of cash flow management versus accruals management on credit rating performance and usage. Review of Quantitative Finance and Accounting, 54, 1163-1193. https://doi.org/10.1007/s11156-019-0 0821-8

\section{Notas}

* Artículo de investigación

Licencia Creative Commons CC BY 4.0

Sugerencia de citación: Correa-García, J., \& Correa-Mejía, D. (2021). Importancia del estado de flujos de efectivo para la gestión financiera sostenible. Cuadernos de Contabilidad, 22. https://doi.org/10.11144/Jav eriana.cc22.iefe 